\title{
Dark Matter Searches
}

\author{
Alessandro Bettini ${ }^{*}$ \\ Dipartimento di Fisica dell'Università and INFN, Padova \\ Via Marzolo 8, 35131 Padova, Italy \\ E-mail: bettini@pd.infn.it
}

I'll start with a summary of the relevant evidence for cold dark matter from astrophysics and cosmology. The Standard Model of subnuclear physics does not have any dark matter particle, but its simplest extensions, the SUSY models, do have a very apt candidate, the neutralino. I will then briefly summarise the status and the expectations for the next years of indirect searches both with earth-based and satellite born particle telescopes and gamma telescopes. I then critically review the main results on the direct searches performed in underground laboratories and the activities to develop the next generation detectors.

European Physical Society

HEP2005 International Europhysics Conference on High Energy Physics EPS (July $21^{\text {st }}-27^{\text {th }}$ 2005) in Lisboa, Portugal

\footnotetext{
${ }^{*}$ Speaker
} 


\section{The Dark Matter Problem. Indirect and Direct Searches for CDM}

The value of the average mass density in the Universe has been determined by a number of observational data, which are relative to very different scales (dimensions) and to different redshifts (epochs), obtained with different techniques, corresponding to different underlying physics. Consistently and independently they provide the same value within the uncertainties. 372000 years (the last scattering epoch) after the initial explosion dark matter shaped the primordial density fluctuations through its gravitational potential. The effects are visible in the spectrum of the anisotropies of the Cosmic Microwave Background (CMB). In particular the height of the first peak in the spectrum gives the total matter density. In general the matter density is sensed at later epochs through its gravitational effects, ranging form the kinetic of the clusters and superclusters of galaxies to the large-scale galaxy correlation function, to the gravitational lensing. Given as a fraction of the critical density the matter density is $\Omega_{m}=0.27 \pm 0.04$.

The contribution of normal matter, called "baryonic" in cosmology, is also determined by independent data sets: from primordial nucleosynthesis, which is a nuclear physics phenomenon at $t=\mathrm{a}$ few seconds and from CMB anisotropy spectrum, which is due basically to electromagnetic processes at a most later epoch. The baryonic density value is only $\Omega_{b}=0.044 \pm 0.04$. We must conclude that the largest fraction of matter is "dark"; its constituents are not baryons and do not have strong neither electromagnetic interactions. We can try to detect them through weak interactions, if they have any. These particles are generically called WIMPs (Weakly Interacting Massive Particles). Evidence for local existence of dark matter comes from the rotation curves of the galaxies, including our own. Dark matter is here, around us, now.

Neutrinos a priori are candidates for dark matter. Not in practice. Indeed cosmology gives a very tight upper limit to the neutrinos contribution and hence to their masses. Neutrinos were already cold at the last scattering epoch and, as a consequence, changing the fraction due to neutrinos of the total dark matter has a very small effect on the CMB spectrum. On the contrary, neutrino mass density has affects the Large-Scale Structures (LSS) formation. Neutrinos, even if non-relativistic, move with speeds larger than the escape velocities from the smaller structures, they freely stream on distances $D_{F}$, which are inversely proportional to the neutrino mass $\left[D_{F}(\mathrm{Mpc}) \approx 1 / m_{v}(\mathrm{eV})\right]$; moving, without interactions, from higher to lower density regions they tend to suppress the formation of structures smaller than $D_{F}$. Data on the LSS show that this effect is small, if any, implying that the fraction of dark matter due to neutrinos to be less than a few percent. Dark matter is predominantly "cold", meaning non relativistic (CDM).

For the virial theorem the average WIMP velocity in the Galaxy frame must be equal to that of the stars, namely $\left\langle\beta_{\chi} \approx \approx 10^{-3}\right.$. The velocity distribution is unknown, but is presumably Maxwellian, truncated at the escape velocity $\left(v_{e s c} \approx 500-600 \mathrm{~km} / \mathrm{s}\right)$. Notice that the typical WIMPs velocities are similar, microscopically, to those of the atomic electrons, a fact relevant for WIMP detection. The expected density is $\rho_{W} \approx 300 \mathrm{TeV} / \mathrm{m}^{3}\left(n \approx 3000 \mathrm{~m}^{-3}\right.$ for $\left.m_{\chi}=100 \mathrm{GeV}\right)$ 
corresponding to a flux $\Phi_{W} \approx 10^{9} \mathrm{~s}^{-1} \mathrm{~m}^{-2}$. Typical kinetic energies are of the order of $50 \mathrm{keV}$ or less.

There are no dark matter candidates in the Standard Model, but several have been proposed by theorists. The most important comes from SUSY, the neutralino, a mixture of the superpartners of the weak bosons and higgs, which is stable if $R$-parity is conserved. Clearly two complementary searches are in order: the search of the CDM in the Universe, to understand the largest fraction of its mass and its artificial production, which will be in the LHC range, for a precise study of their properties.

I'll limit the discussion to neutralinos $(\chi)$ in this review. Neutralinos are Majorana particles. They can be detected via their mutual annihilation in the "indirect" searches and via their elastic scatters on nuclei in the "direct" searches.

Notice that neutralino is only one of the foreseen and not foreseen possibilities, we are really hunting in the dark. We must tackle the dark matter problem with a set of complementary approaches: experiments at LHC, direct searches and indirect ones by means different messengers.

\section{Indirect Searches, on Ground and in Space}

Indirect searches look for the high-energy particles produced by the neutralinos annihilation or for the daughters of those particles. Neutralinos tend to decay in the largest mass particles; hence, depending on the mass, they would decay in $\tau$ leptons, $b$ and $t$ quarks, $W$ and $Z$ bosons.

The signatures are the following:

- non-standard flux from a localised source. The idea is that WIMPs can become trapped in the Sun or in high-density sites like the Galactic Centre (GC). Annihilation rate is proportional to the square of neutralino density. To point back to the source the messengers must be neutral: photons or neutrinos.

- monochromatic photons coming from the processes $\chi \chi \rightarrow \gamma \gamma$ or $\chi \chi \rightarrow \gamma Z$ from localised or even from diffuse sources;

- distortions in the energy spectrum of photons or "rare" particles such as positrons and antiprotons. Consider as a relevant example the $\gamma$ ray spectrum shown in Fig. 1. The most important standard contribution is the decay of the $\pi^{\circ}$ 's produced in the collisions of cosmic protons and nuclei. The resulting $\gamma$ spectrum has a (Jacobian) peak at $m_{\pi} / 2$ and decreases with a power law at higher energies, a line in a logarithmic plot. WIMPs contribution appears as a hump of characteristic shape, above that line, at several $\mathrm{GeV}$ energy, dependent on the WIMP mass $m_{\chi}{ }^{[1]}$. Such an excess from the GC was reported already in 1998 by EGRET $^{[2]}$. But this sole observation could not exclude more mundane interpretations. Clearly, more complete data are needed to establish the presence of CDM. 
These data may well come from the next generation of telescopes and spectrometers. Spectrometers on satellite will be focussed on charged particles (PAMELA ${ }^{[3]}$ and AMS $^{[4]}$ ) and on gammas $\left(\mathrm{GLAST}^{[5]}\right)$. The gain in angular and energy resolution and in acceptance are such that qualitatively new results can be expected.

Three new gamma-ray Cherencov telescopes on Earth (CANGAROO $\mathrm{II}^{[5]}$ in Australia, H.E.S.S. ${ }^{[6]}$ in Namibia and MAGIC ${ }^{[7]}$ in La Palma) are taking data that show already a wealth of new sources and phenomena; the construction of a fourth, VERITAS ${ }^{[8]}$ in Arizona is progressing. Their energy thresholds are now as low as $50-100 \mathrm{GeV}$, lower than the upper limits of the new space telescopes such as GLAST, allowing overlapping observations.

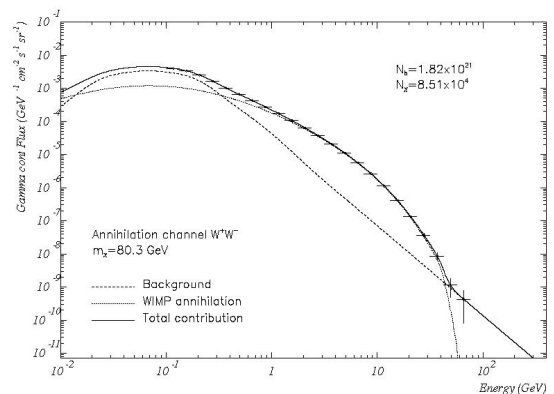

Fig. 1. Simulation of the result of two years data from GLAST, assuming the excess of EGRET to be a signal from WIMP annihilation ${ }^{[1]}$

In conclusion, with these instruments gamma-ray astrophysics will certainly produce extremely interesting data in the next decade, possibly surprises, hopefully contributions to the understanding of dark matter.

The muon neutrinos astrophysics has already started with large-volume underground observatories (SuperKAMIOKANDE ${ }^{[9]}, \mathrm{MACRO}^{[10]}$, Baksan $^{[11]}, \mathrm{AMANDA}^{[12]}$ ) detecting the $\mu$ 's produced by neutrino collisions and searching for localised sources. In this case atmospheric neutrinos are the, rather well known, background. Up to now no signal was detected, due to the still too small size of the detectors, but the cubic kilometre size observatories, ICECUBE under construction at the South Pole and a possible KM3 in the Mediterranean Sea, may well reach sensitivity to dark matter.

\section{Direct Searches. General Principles}

The direct searches look for the WIMPs elastic scattering detecting the energy deposited by the recoiling nucleus.

The interaction cross sections of the neutralinos can be calculated on the basis of SUSY models, but several parameters of the theory being unknown, there are large uncertainties in the results $^{[13]}$. Two basic possible couplings can be distinguished:

- "spin-dependent" (SD). It is an axial vector coupling to the nucleons spins; only unpaired nucleons couple. 
- "spin-independent" (SI). It is a scalar interaction with the mass of the nucleons; if it is coherent it is proportional to $A^{2}$. Heavier nuclei are clearly at advantage, but one must be careful because the coherence is lost at momentum transfer $(Q)$ larger than the inverse radius. The cross section decreases with increasing $Q^{2}$, more sharply for heavier nuclei, a behaviour parameterised in the "form factor". This is a function of $Q^{2}$ of fundamental importance for the interpretation of the experimental data that must be experimentally determined for the relevant nuclides in the relevant recoil energy range. Still, the use of the form factors is a source of uncertainty when comparing experiments on different nuclides.

The WIMPs flux is uncertain too. One must assume a definite halo model, namely a set of hypothesis of the local WIMP density and velocity distribution, in which the solar system moves. The expected rates are really very small. The differential rate is the counting rate per unit detector mass, exposure time and energy range; I'll use as unit the dru $\equiv 1 \mathrm{count} /(\mathrm{kg} \mathrm{keV} \mathrm{yr})$.

The results of the calculations and of the experiments are usually represented in the plane of the cross-section, reduced to a single nucleon, $\sigma_{p}$ vs. $m_{\chi}$. This corresponds to projecting on a plane the multidimensional parameter space (local $\chi$ density, SI and SD cross section ratio, ratio of SD coupling to neutrons and to protons, local velocity, etc.). A necessary but ambiguous operation.

There is no firm theoretical upper limit on $m_{\chi}$, but one can take reasonably $1000 \mathrm{GeV}$; a lower limit at $\approx 50 \mathrm{GeV}$ is given by LEP, but it is model dependent. The theoretical expectations for the cross sections have even more uncertainties, ranging from $10^{-5}$ to $10^{-12} \mathrm{pb}$. The corresponding rates depend on target nucleus and on energy threshold. As an indication, they range from 1 to $10^{-7}$ dru. The "ultimate" detector should have a sensitive mass of the order of $100 \mathrm{t}$ with a background rate smaller than a few counts per year in a $10 \mathrm{keV}$ window at, say, 30 $\mathrm{keV}$. This is impossible today, but the progress of the field is impressive.

Experimentally the target medium is also the detector, in which one tries to detect the nucleus elastically hit by the WIMP. The energy is deposited in three forms: as ionisation charge, scintillation light or phonons.

The small fraction of the deposited energy going to ionisation, at these small velocities, is not described by the familiar Bethe-Block theory, as discussed by Lindhard ${ }^{[14]}$. It is a function of energy depending on the nucleus and on the applied electric field. One defines the "quenching factor" an energy dependent quantity, which must be determined experimentally. The response of the detector is first measured with a $\gamma$ calibration source of energy, say, $E_{e e}$ ("electron equivalent energy" measured in " $\mathrm{keV}_{e e}$ "). Then the recoil energy that gives the same response $\left(E_{r e c}\right.$ in $\left.\mathrm{keV}_{r e c}\right)$ is determined. The quenching factor is defined as $Q_{F}=E_{e e} / E_{r e c}$. Typical values range from 0.05 to 0.30 .

The experimental challenges are a very small signal rate, small energy deposits (several $\mathrm{keV}$ ) and a signal spectrum decreasing with increasing energy, as all backgrounds do. Not only the detectors must be operated in an underground laboratory, but specific R\&D is needed to achieve the highest radiopurity of the screening materials and of the detector itself. 
We can distinguish three basic backgrounds: 1. electromagnetic, due to $\gamma$ 's and $e$ 's; it's the dominant one; 2. radioactive contaminants of the surfaces, which release only part of the energy in the sensitive volume, simulating WIMP signal; 3. neutrons, which give nuclear recoils indistinguishable from WIMPs. The first background can be discriminated by measuring different fractions of the recoil energy: phonons, photons and free electrons.

For optimal background control the discrimination should be on event-by-event basis and not merely statistical.

\section{Direct Searches Looking for Modulation}

One of the experimental methods in the CDM search is to look for characteristic signatures not easily reproducible by the backgrounds. The solar system moves in the WIMPs halo with a velocity $v_{0} \approx 220 \mathrm{~km} / \mathrm{s}$ (with large uncertainties). The Earth orbital velocity is in the same direction in June, in opposite direction in December. As a consequence a small $(< \pm 7 \%)$ modulation of the WIMPs flux and of the counting rate is expected ${ }^{[15]}$. The signal has the following characteristics: the rate should be modulated with a sinusoidal function of the time with maximum around June $2^{\text {nd }}$ and amplitude of a few percent. Moreover, the modulation should be present only at very low energy.

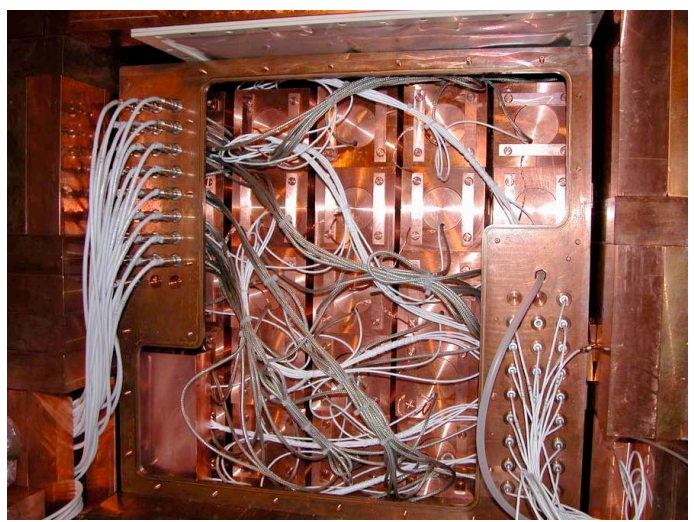

Fig. 2. The LIBRA set-up of DAMA presently running

The DAMA experiment (see Fig. 2 for the presently running set-up) at the Gran Sasso Laboratory of the INFN (LNGS) was designed to this aim. It consists of 9 hyper-pure NaI crystals, for a total sensitive mass of about $100 \mathrm{~kg}$, each equipped with two low-background photomultipliers. The software threshold is $2 \mathrm{keV}_{e e}$ (corresponding to $10-15$ photoelectrons). The background at threshold is about 0.5 dru. The experiment, now completed, ran for 7 years (1996-2002) continuously (after the first), with monitoring and control of the long-term stability of the relevant experimental parameters.

The total exposure has been of $107731 \mathrm{~kg} \mathrm{~d}$. The final results have been published ${ }^{[16]}$ since two years and are summarised in Fig. 3 that shows the time dependent part of the rate, folded on one year. All the expected signal characteristics - modulation period and phase, 
sinusoidal behaviour, amplitude of a few percents - are present in the lowest energy bins, while no modulation is seen at higher energy nor when more than one detector is hit (multiple hits). There are several natural and artificial phenomena that have one-year period, but the group reports to have carefully checked all possible sources of fake modulation and found none.

The DAMA result gives model independent evidence. The calculation of an allowed region in the $\sigma$ vs. $m_{\chi}$ depends instead on several assumptions. I'll discuss this point at $\S 6$.

Clearly, the result must be checked by an independent experiment with better or equal sensitivity. Given the uncertainty in the couplings, the same nuclei (NaI) should be used. The DAMA group itself is now running, since 2003 , the LIBRA ${ }^{[17]}$ set-up with a NaI mass increased to $\approx 250 \mathrm{~kg}$.
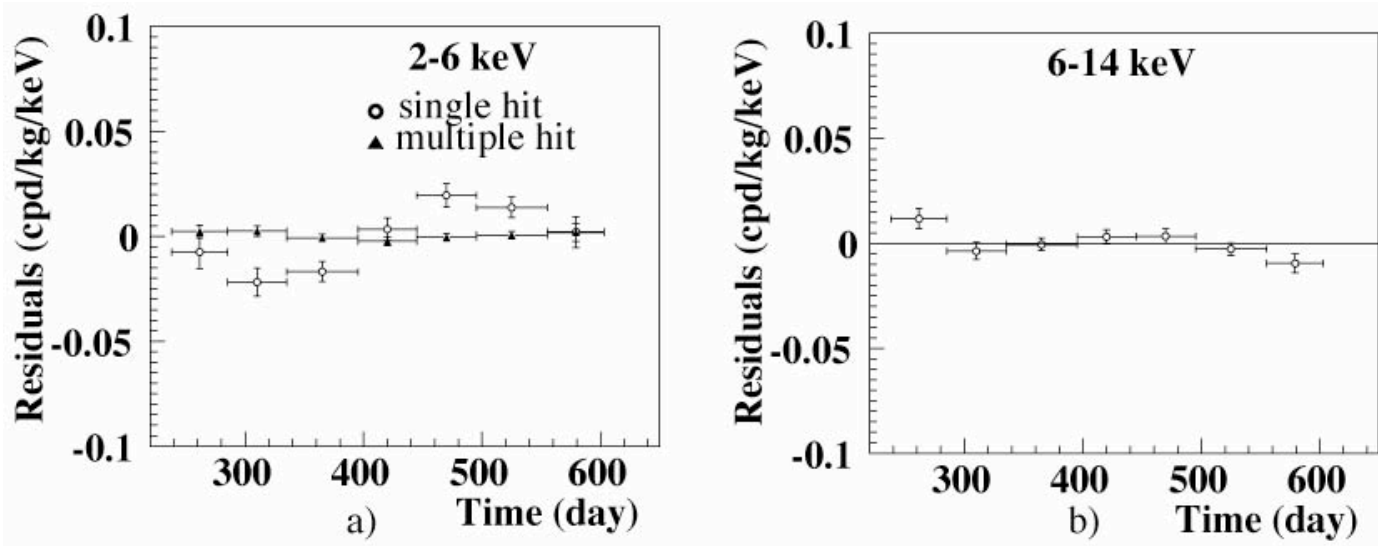

Fig. 3. DAMA time-dependent part of the rate. a) $2<E_{r e c}<6 \mathrm{keV}_{e e}$ single hit and multiple hit; b) $6<E_{\text {rec }}<14 \mathrm{keV}_{e e}$

A fully independent experiment, ANAIS ${ }^{[18]}$, is under development at the Canfranc Underground Laboratory (LSC). $14 \mathrm{NaI}$ crystals $10.7 \mathrm{~kg}$ mass each are stored underground since 1988 (to get rid of the cosmogenic activation). A first prototype made with one crystal seen by one PMT, hence with a $4 \mathrm{keV}$ threshold (too high for physics experiment), has shown a background index $b=1 \mathrm{dru}$, a value that should make the experiment possible. A second prototype with two PMTs ( $2 \mathrm{keV}$ threshold possible) and better screening is being developed. It is extremely important that the ANAIS experiment becomes operational as soon as possible, being the only one capable to confirm or dispute DAMA. It will profit of the new structures of the Laboratory, a heritage to the community that Angel Morales left us as the realisation of his, unfortunately last, dream.

In principle, another characteristic signature of WIMPs is the annual variation of the recoil direction. But its measurement can be done only in a low-pressure gas, a technique limited to small sensitive masses. 


\section{Direct Searches with Cryogenic Techniques}

All the other experiments follow a different method. In consideration of the fact that the residual backgrounds cannot be accurately modelled and subtracted one must develop selection criteria to define, preferably with blind decisions, a region in the experimental parameter space that should be "background free". One then assumes a definite theoretical framework (an halo model, a type of coupling, etc.) and calculates for each $m_{\chi}$ the maximum possible signal rate compatible with the data. The result is an "exclusion curve" in the $\sigma_{p}$ vs. $m_{\chi}$ plane.

Three experiments, CDMS at the Soudan Laboratory, EDELWEISS at LSM and CRESST at LNGS, employ crystal bolometers at millikelvin temperatures. All measure the "heat" i. e. the thermal excitation, but CDMS in the form of not-yet-thermalised phonons, the others by the temperature increase $\Delta T=E_{\text {rec }} /(C M)$, i. e. thermalised phonons. All measure a second quantity: CDMS and EDELWEISS the ionisation, CRESST the scintillation light. The ionisation-to-heat and light-to-heat ratios being much larger for electron recoils than for nuclear recoils, provide a powerful discrimination of the electromagnetic background.

Athermal phonons detected by CDMS still remember their geometrical origin and are used to discriminate the $\gamma$ interactions on the surface, which give an earlier and faster rising pulseshape. CRESST tries to get rid of surface events just avoiding surface electrodes and reading out the light.

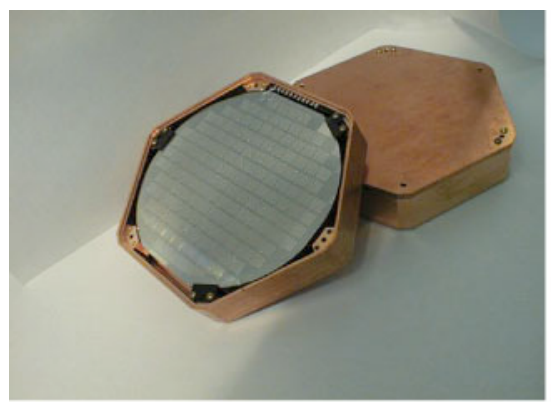

a)

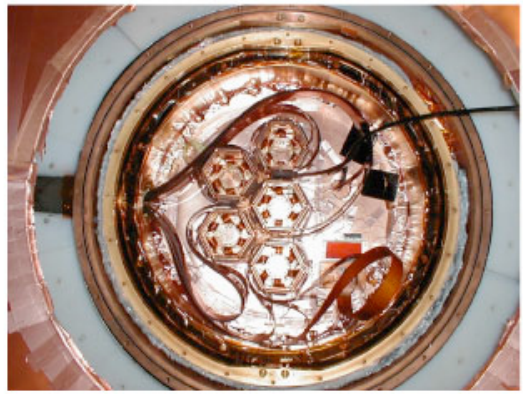

b)

Fig. 4 a) a ZIP detector; b) five towers in the cryostat of CDMS

Neutron background is almost indistinguishable from the WIMP signal and must be avoided by shielding and accurate screening of the materials.

CDMS elementary detectors, called ZIPs (Fig. 4a), are assembled in structures called towers. A tower contains Ge ZIPs and Si ZIPs. WIMPs interactions are expected on Ge not on $\mathrm{Si}$, while neutrons interact with both at similar rates. The comparison gives a handle to monitor the neutron background. Another difference is that WIMP interactions are single-site, neutron can be multiple-site. A first run in 2004 (not shown here) with 4 Ge and 2 Si ZIPs, with an exposure of $19.4 \mathrm{~kg} \mathrm{~d}^{[19]}$, observed 0 events in the blindly defined signal region $\left(10<E_{e e}<100\right.$ $\mathrm{keV}$ ), a second in 2005 with a second tower with 2 Ge and 4 Si ZIPs and an exposure of $38 \mathrm{~kg}$ $\mathrm{d}^{[20]}$, observed one event (Fig. 5a).

The exclusion curves are shown in Fig. 5b. CDMS is presently the most sensitive experiment due to its being (almost) background free, not so much to the size of its exposure. 

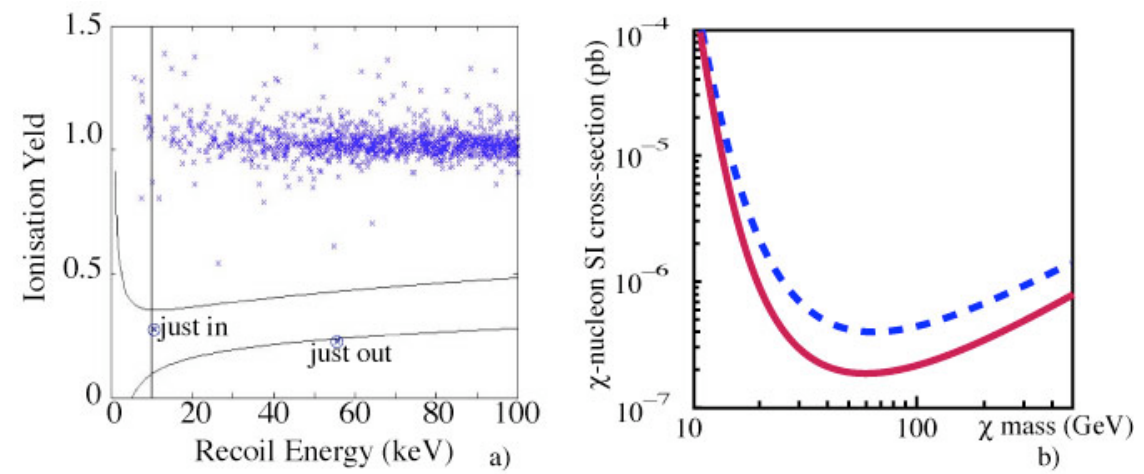

Fig. 5. a) Ionisation vs. recoil energy in CDMS05; b) exclusion curves of CDMS: 2004 dotted, 2005 continuous

CDMS has now installed 5 towers (Fig. 4b) for a total of $4.75 \mathrm{~kg}$ of Ge and $1.1 \mathrm{~kg}$ of Si; it will run through 2006, improving the sensitivity by an order of magnitude. Further improvements are being developed to reduce the backgrounds and increase the sensitive mass in a number of stages aiming to the ton scale; this, if background discrimination is improved by four orders of magnitudes for the final scale, should allow sensitivities in the range of $10^{-10} \mathrm{pb}$. Super-CDMS will need to operate in an underground laboratory much deeper than Soudan, where is presently located, which has only a $780 \mathrm{~m}$ deep overburden.

EDELWEISS $1^{[21]}$ concluded with an exposure of $62 \mathrm{~kg} \mathrm{~d}$. It is based on three Ge detectors at $10 \mathrm{mK}$ temperature with a total mass of about $1 \mathrm{~kg}$. In the "signal window", similar to that of Fig. 5a), the experiment observed 53 events. The spectrum of this sample is shown in Fig. 6a) together with the expectations for WIMPs with $\sigma=10^{-5} \mathrm{pb}$ and different masses.
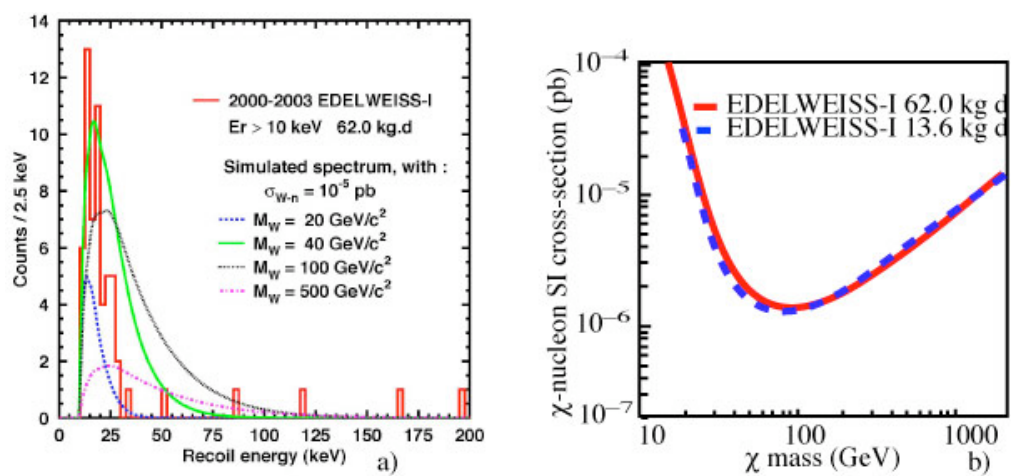

Fig. 6 a) Energy spectrum for the sample in the signal region of EDELWEISS1; b) the exclusion curves

The authors state that the spectrum is incompatible with a single WIMP and that complementary data indicate that these events are probably due to surface electrons and to neutrons. This is presumably true, but one may observe that the spectrum is fully compatible with a combination of a fraction of background (whose shape is unknown) and of WIMP signal (e.g. $m_{\chi}=20 \mathrm{GeV}, \sigma \approx 10^{-5} \mathrm{pb}$ or $m_{\chi}=40 \mathrm{GeV}, \sigma \approx 0.5 \times 10^{-5} \mathrm{pb}$ ). These values are fully compatible 
with the DAMA result, but obviously can neither confirm neither disprove it. The authors in the calculation of the exclusion curve (continuous one in Fig. 6b) use both the number of events and the shape of the spectrum, but they assume the last to be signal only not allowing for an arbitrary mixture of signal and background. This procedure pushes the limit towards lower values. Nonetheless Fig. 6b shows no improvement in the latest results compared to the previous ones with quadruple exposure; a fact due to the presence of background.

EDELWEISS2 will run initially with 28 Ge detectors with a sensitive mass $\approx 10 \mathrm{~kg}$ and a number of improvements: athermal phonon detection (to discriminate surface events), installation of a $\mu$ veto, an even more demanding material screening, etc. It will be commissioned in 2006 . The $2^{\text {nd }}$ phase is planned for 110 detectors corresponding to $\approx 40 \mathrm{~kg}$ sensitive mass aiming to a sensitivity of $10^{-8} \mathrm{pb}$.

CRESST2 ${ }^{[22]}$ ran with two $\mathrm{CaWO}_{4}$ crystals, $250 \mathrm{~g}$ each, at $6 \mathrm{mK}$ temperature. The phonon signal (heat) and the scintillation light signal are read out to discriminate the electromagnetic background. Neither a $n$-shield nor a $\mu$-veto had been installed in this first run. The preliminary measurements necessary for such a new detector concept have been done, those, in particular, of the quenching factors for $\mathrm{Ca}, \mathrm{W}$, and $\mathrm{O}$. For tungsten the quenching factor is very small, $Q_{F}=0.025$.

The "signal region" is defined in the Light-yield vs. Phonon-energy plot shown in Fig. 7a). This definition depends on the assumption on whether WIMPs scatter on all nuclei (between dotted lines) or on the $\mathrm{W}$ only (below continuous line). In a $20.5 \mathrm{~kg} \mathrm{~d}$ exposure, 18 events are seen overall in the two detectors in the first hypothesis, 3 in the second. The 3 events are all in one counter (upper panel in Fig. 7a); the authors observe that this is the one with worst resolution in the light channel, a fact that facilitates the electron recoils to leak into the signal region. If one decides (somewhat arbitrarily) to assume scattering on W only and the "good" detector only one ends up with zero background and the dotted exclusion curve of Fig. 7b). If one takes all the data, the curve is the continuous one. Again the importance of being background-free is evident.
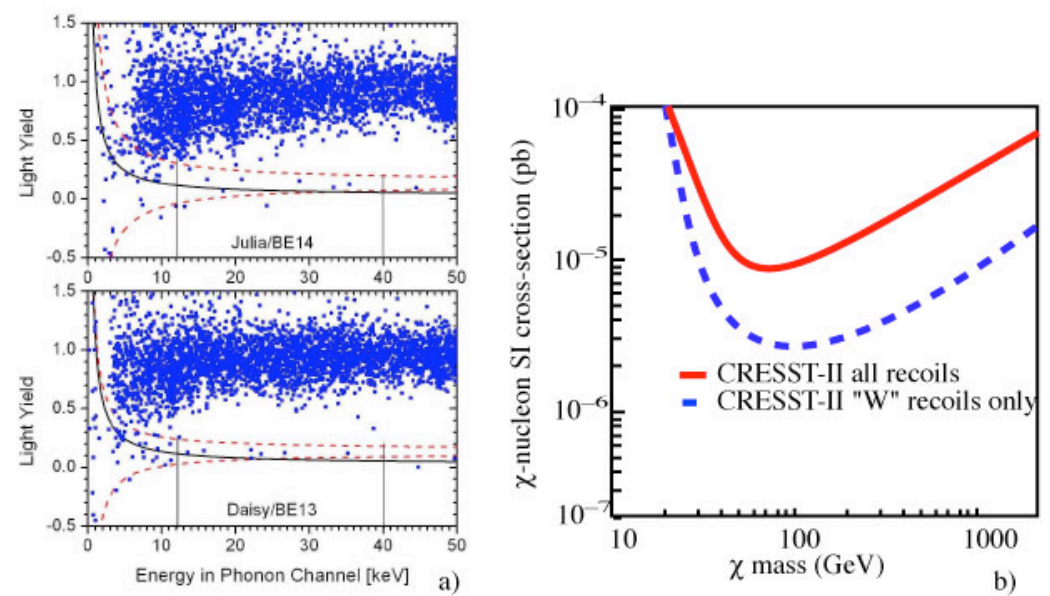

Fig. 7 a) Light vs. heat for two CRESST2 detectors. b) Exclusion plots 
The next phase of CRESST2 is already started. The shields against neutrons, dominating the background in the $1^{\text {st }}$ phase, has been installed, the $\mu$ veto too. Discrimination criteria of $n$ from $\chi$ on the basis of their different cross sections ratios on $\mathrm{O}$ and $\mathrm{W}$ are being developed. By the end of 2005 a $3 \mathrm{~kg}$ detector mass should be ready, increasing later to $10 \mathrm{~kg}$ with an expected sensitivity of $10^{-8} \mathrm{pb}$ at $m_{\chi}=60 \mathrm{GeV}$.

Fig. 8 shows a summary of the results (including ZEPLIN1 to be discussed in §6). As already recalled, the parameters space is multi-dimensional and its projection on $\sigma$ vs. $m_{\chi}$ plane can be done only within a given framework of assumptions on the other parameters. In particular, the $\chi$-nucleus couplings can be very different for different isotopes. Fig. 8 assumes purely SI couplings. I hand-drew the curves without extreme care on the precision, because they are meant to be only indicative. The DAMA ${ }^{[16]}$ regions are those reported by DAMA itself, which are different by those reported by other experiments quoting DAMA. The region above $200 \mathrm{GeV}$ (light colour) is for a very low value of the local velocity, $170 \mathrm{~km} / \mathrm{s}$. Regions at lower cross section values are possible if a fraction of SD coupling is present.

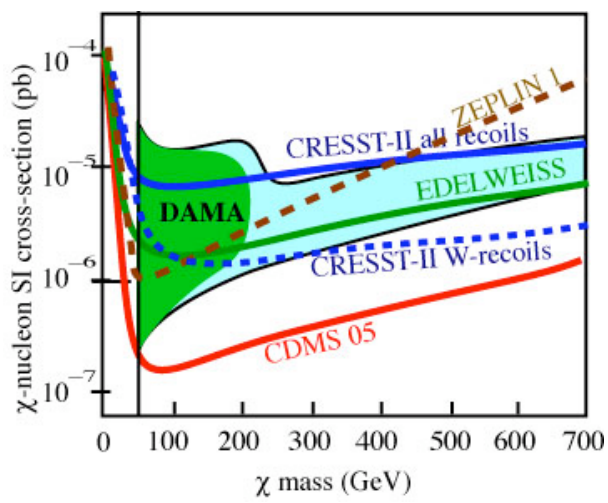

Fig. 8. Hand-drawn comparison amongst the results

The superior CDMS sensitivity shows clearly the importance of being background free. CDMS appears to disprove DAMA, but the target nuclei are different, the contributions of SD and SI interactions may be different, the interplay of the form factors is different, etc. The other experiments are limited by background.

To complete the comparison the indirect search results must be mentioned. The relevant data are the limits of an excess $\mu$ flux from the Sun from SuperKAMIOKANDE ${ }^{[9]}$ and Baksan $^{[11]}$. These long-term exposures are important for the spin-dependent $\chi$-proton coupling (Sun is made of protons). For this they are two orders of magnitudes more sensitive than direct searches. C. Savage et al. ${ }^{[23]}$ performed a complete analysis considering both direct (not including CDMS 2005, not yet available at that time) and indirect searches data and assuming both SD and SI couplings. Within their hypothesis, the conclusion is that there is only a region in which DAMA is compatible with the other data, at small masses for $5<m_{\chi}<15 \mathrm{GeV}$. 


\section{Noble Fluids}

Noble liquids, Ar, $\mathrm{Xe}$ and $\mathrm{Ne}$, are very promising substances for the next generation detectors for WIMPs search. After several years of R\&D, massive detectors are now in the design or construction phases. The attractive characteristics of noble liquids are:

- can be easily assembled in large masses, up to hundreds tons, at reasonable costs;

- techniques to purify large quantities of such liquids at extreme radiopurty levels are available, as those developed by BOREXINO;

- self-shielding structures can be built, with the central fiducial mass shielded by the external one without any surface between them (avoiding possible backgrounds);

- the external mass can be instrumented and used as an active veto ( $\mu$, anti-Compton, etc.)

The exploitation of the physical process of energy transfer to the medium from the recoil electrons on one side and nuclei on the other gives two independent means to discriminate the dominant electromagnetic background. They are based on the detection of the ionisation and of the scintillation light, as follows.

- Part of the energy deposited by an ionising particle in these media is finally released as scintillation light emitted by excited dimers $\left(\mathrm{Xe}_{2}{ }^{*} \rightarrow 2 \mathrm{Xe}+\right.$ photons; $\mathrm{Ar}_{2}{ }^{*} \rightarrow 2 \mathrm{Ar}+$ photons). The de-excitation curves have two components: one fast from the singlet ${ }^{1} \Sigma$ states, which are populated mainly by the heavy recoils, and one slow from the triplet ${ }^{3} \Sigma$ states, populated by the light recoils. This behaviour has been discovered and studied several years ago by T. Doke and collaborators ${ }^{[24]}$.

- The ratio of ionisation to scintillation is much smaller for the slow-moving nuclear recoils than for electrons. The few ionisation charges can be detected by the proportional scintillation produced in the very strong electric field near to a thin anode wire. The method was proposed and shown to work in liquid Xenon by P. Picchi and coll. $^{[25]}$ and later improved ${ }^{[26]}$ with the idea of the dual-phase detector. As shown schematically in Fig. 9, a uniform electric field is applied to drift the electrons, produced in the liquid, vertically up to the surface and extract them into the gas phase (with almost 100\% efficiency); here they are amplified using appropriate grid structures and the proportional scintillation light is detected. In conclusion, two light signals are detected, a prompt one, $S_{1}$ proportional to the primary scintillation light, and a delayed one, $S_{2}$ proportional to the ionisation. Their ratio $S_{2} / S_{1}$ discriminates electrons from nuclear recoils.

A few considerations are in order. The ionisation electrons are very few and must be collected with high efficiency; in particular, the electric field must be accurately designed, in order, for example, not to "lose" electron in the non-uniformities close to the race-track. Photons must be efficiently collected too, because they are so few (e.g. $\approx 200$ at $16 \mathrm{keV}$ in Xe). Another important aspect is the resolution in the localisation of the event, which allows the exclusion of "dirty" regions. It can be achieved building the detector as an image chamber or using arrays of photomultipliers. 
Pioneering work on LXe as a WIMP target was done by DAMA ${ }^{[27]}$ and then by the, already mentioned, ZEPLIN1 experiment at the Boulby mine. Notice that Xe has several stable isotopes, five with more than $9 \%$ abundance, ranging in mass from $A=129$ to $A=136$, with different form factors and different spins.

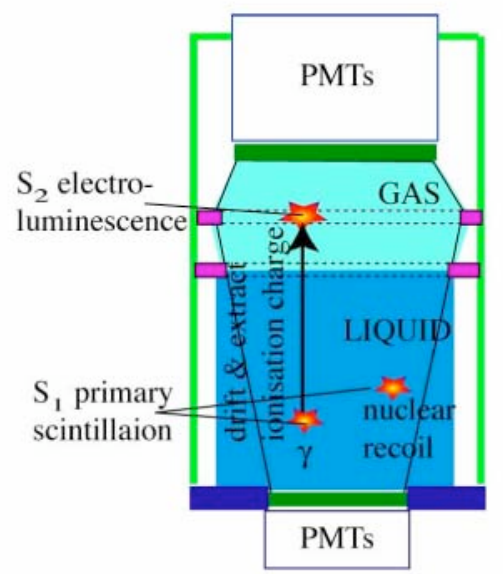

Fig. 9. Principle of the dual-phase detectors

ZEPLIN1 had a fiducial mass of $3.2 \mathrm{~kg}$ of LXe, contained in a copper cryostat equipped with three photomultipliers, which was enclosed in an active scintillator veto. The light signal gives a rough energy measurement with $100 \%$ uncertainty at the $2 \mathrm{keV}_{e e}$ threshold. The discrimination against the electromagnetic background is based on the above-mentioned difference in the dimers decay times, which are not very different for Xe (4.3 ns and $22 \mathrm{~ns}$ ); as a consequence, the discrimination could be only statistical (as opposed to event by event) and based on the leading edge distribution of the signal. To be safe this procedure needs a very good knowledge and control of the systematic uncertainties, to be obtained with calibration runs in situ.

ZEPLIN $1^{[28]}$ published the results for a $293 \mathrm{~kg} \mathrm{~d}$ exposure which are reproduced in Fig. 8. Unfortunately the result is weakened by the following circumstances: calibration runs have been performed only on the surface and not in situ and the light decay constant ratio was assumed to be energy independent.

Several projects using noble liquids are in different stages of development. Some use liquid-phase only: CLEAN with LNe, XMASS at Kamioka with LXe; more employ the doublephase concept: ZEPLIN 2 and 3 at Boulby and XENON at LNGS with Xe, WARP at LNGS and DEAP in R\&D at Los Alamos with LAr. I'll discuss the most advanced of them now.

XMASS is a Japanese project for multipurpose detectors based on large Xe masses, both single-phase and double-phase.

The one-phase XMASS ${ }^{[29]}$ project will be a spherical envelope completely surrounded by photomultipliers containing $800 \mathrm{~kg}$ of LXe (Fig. 10, right), enclosed in a standard external shield. The inner fiducial $100 \mathrm{~kg}$ mass is shielded by the outer liquid. The spherical geometry of 
the PMs allows a fairly accurate vertex reconstruction. With the design energy threshold $E_{t h}=5$ $\mathrm{keV}_{\mathrm{ee}}$ the experiment should reach in a $0.5 \mathrm{t}$ yr exposure a sensitivity of a few $10^{-9} \mathrm{pb}$. This large mass detector can be employed for annual modulation search too, with sensitivity of $10^{-8}$ $\mathrm{pb}$ at $m_{\chi}=60 \mathrm{GeV}$ and $10^{-7} \mathrm{pb}$ at $m_{\chi}>200 \mathrm{GeV}$. Experience with different prototypes is going on at Kamioka.
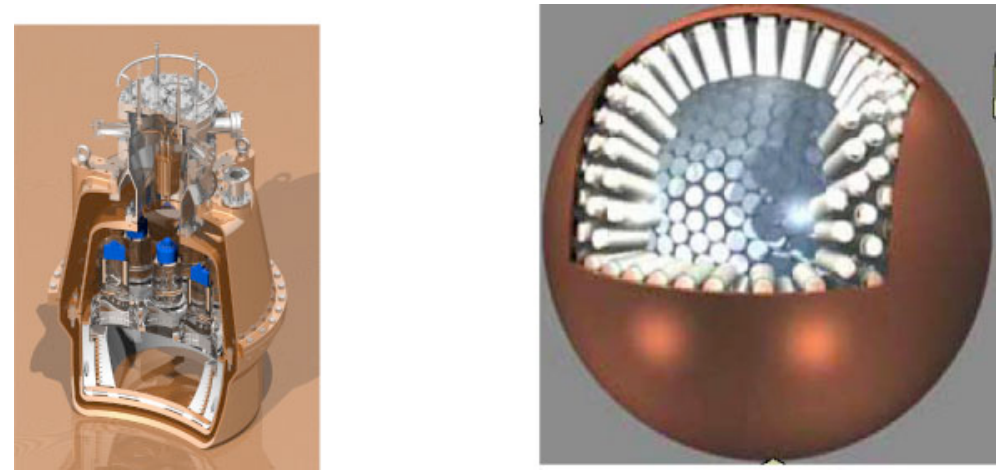

Fig. 10. Cut-away views: of ZEPLIN 2 (left) and of XMASS 800 (right)

A $15 \mathrm{~kg}$ module is the prototype of the XMASS dual-phase project ${ }^{[26]}$. Data have been collected with calibration sources of $22 \mathrm{keV} \gamma$ s and neutrons (to induce Xe recoils). In the $S_{2}$ vs. $S_{1}$ plot $\gamma$-ray population appears at $S_{2}$ values much larger than the Xe-recoils population, as expected. But a third, smaller population is present between the two, presumably due to small contamination of structural elements as wires, racetracks, etc. and/or on the surfaces as the light reflecting foil. These background "tails" are extremely relevant as those that limit the rejection power. If they are small enough, rejection power $>1000$ should be possible but the real value must be found experimentally.

The next steps with LXe dual-phase detectors at Boulby are ZEPLIN 2 and 3, two complementary detectors. The long-range programme aims the ZEPLIN MAX, $1 \mathrm{t}$ sensitive mass detector with $10^{-10} \mathrm{pb}$ sensitivity.

ZEPLIN 2 (Fig. 10 left) has been just installed underground. With its $30 \mathrm{~kg}$ fiducial mass its aims to $10^{-8} \mathrm{pb}$ sensitivity. Ionisation electrons produced in the LXe are drifted on a $10 \mathrm{~cm}$ distance, extracted in the gas phase and amplified in a $5 \mathrm{~mm}$ proportional scintillation gap. A set of PMs above the gap measures the two light pulses. The ZEPLINs do not use an outer LXe volume as a shield. Rather, the active veto is liquid scintillator in a second container, introducing a copper surface at the limit of the sensitive volume. The mass of ZEPLIN 3 is smaller, $8 \mathrm{~kg}$, but the detector is designed for a superior light collection capability, having the PMs immersed in the liquid, and a precise 3D reconstruction in the gas.

The US XENON ${ }^{[30]}$ project has been approved by NSF and LNGS. It is a modular design of $100 \mathrm{~kg}$ LXe mass units. This unit is a 3D position sensitive TPC, providing full vertex reconstruction. The outer LXe is employed as an active veto.

Preliminary measurements have already been done, including those of the quenching factor in the relevant energy range, at $15 \mathrm{keV}_{\text {rec }}$ and $50 \mathrm{keV}_{\text {rec }}$. Tests on the surface have shown 
a recoil-gamma discrimination with $S_{2} / S_{1}$ by a factor 300 (limited, again, by the tails of the $\gamma$ distributions). A $10 \mathrm{~kg}$ prototype, XE-10 (see Fig. 11, left) will be installed at LNGS in 2006; the $1^{\text {st }}$ XENON-100 module should be ready by 2007 , aiming at a background index $b<10^{-4}$ dru, which will allow a background-free three month run. The projected sensitivity is a few $10^{-9} \mathrm{pb}$.
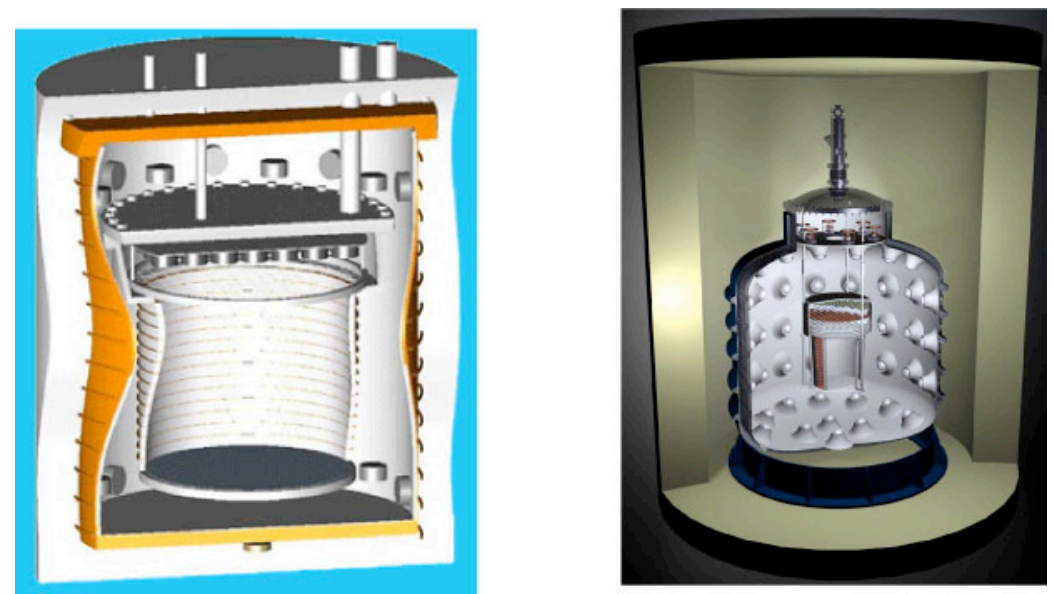

Fig. 11. Left. The XE-10 prototype of the XENON experiment; Right. The 1001 module of the WARP experiment

WARP $^{[31]}$ is a dual-phase LAr project proposed by C. Rubbia and collaborators and approved at LNGS. Ar compared to Xe has advantages and disadvantages: its nucleus is lighter, losing for SI coupling roughly a factor $A_{A r}^{2} / A_{X e}^{2}=1 / 10$; on the other hand Ar nucleus is smaller, hence its form factor decreases slower with increasing momentum transfer, allowing a higher experimental threshold. The big disadvantage is the presence of the $\beta$-active ${ }^{39} \mathrm{Ar}$ with an activity of about $1 \mathrm{~Bq} / \mathrm{kg}$; the big advantage is the slow/fast scintillation lifetime ratio, which is as large as $1.6 \mu \mathrm{s} / 7 \mathrm{~ns}$.

The first module (see Fig. 11 right) will be a $140 \mathrm{~kg}$ fiducial volume LAr TPC surrounded by a contiguous active veto $60 \mathrm{~cm}$ thick. The inner volume will be optically separated by a highly reflective film with wave-length shifter and seen by a fine grain PMs array providing high light collection efficiency. In order to be background-free in its $30-100 \mathrm{keV}$ energy window in a, say, one year exposure the background index must be $b<3 \times 10^{-7}$ dru, corresponding, in particular, to a rejection of the dominant ${ }^{39} \mathrm{Ar}$ contribution by a factor $10^{8}$. Simulations show that this is within reach with the two independent discriminating ratios: fast/slow and $S_{1} / S_{2}$. The caveat is that the ${ }^{3} \Sigma$ and ${ }^{1} \Sigma$ lifetimes have been measured so far with $\alpha$ particles and nuclear fragments at the $\mathrm{MeV}$ energy scale. The extrapolation to several $\mathrm{keV}$ energy slow-moving nuclear recoils should be controlled experimentally. Moreover, the quenching factor and its energy dependence have not been measured yet. A $2.3 \mathrm{~kg}$ mass prototype is presently running at LNGS with very encouraging preliminary results. 


\section{Conclusions and Outlook}

The fundamental research for dark matter is a very active field, with many complementary running experiments and novel techniques under development. Only one experiment, DAMA, searches for the characteristic signal of annual modulation and it only reported positive evidence. Only one experiment, CDMS is background free. It gives negative evidence at a level difficult to reconcile with DAMA, but no firm, model independent conclusion can be drawn. The theoretical uncertainties are so large, the potential differences between the effects on different nuclei so important, that only a modulation sensitive, independent, $\mathrm{NaI}$ experiment can confirm or disprove the DAMA observation; ANAIS is a candidate.

Experiments not looking for modulation have reached sensitivities $\left(10^{-6}-10^{-7} \mathrm{pb}\right)$ in the region of the most optimistic SUSY predictions; substantial progress is expected for the next years.

R\&D of a new generation of experiments, based on dual-phase LXe and LAr, shows extremely promising perspectives, but the commissioning underground of 10-100 kg detectors has just started. Background-free exposures in the $10 \mathrm{t}$ yr range may lead, in absence of limits due to systematics, to $10^{-10} \mathrm{pb}$ scale, exploring the largest fraction of the parameters space.

But SUSY is only a possibility and we should prepared to surprises. We must then rely on a set of complementary, but co-ordinated, experimental approaches. Beyond the direct searches in the underground laboratories, LHC will soon produce data on the existence or not of SUSY particles and, if they exist, on their nature, new observatories in space and on ground are giving and will give astrophysical data of unprecedented quality and quantity. Only with a joint development of our understanding of the microcosm and the macrocosm we can hope to solve the dark matter(s?) problem.

\section{Acknowledgments}

I'm grateful to the organisers and in particular Gaspar Barreira and to Jose Bernabeu for their kind invitation to give this review within a splendid scientific programme; to them and to Joao Martins and Natalia Antunes for the beautiful organisation of the conference.

In the preparation of this papers I have been helped by several people working in the experiments; I'm inedited in particular to E. Aprile, L. Baudis, R. Bernabei, A. Deangelis, S. Gentile, G. Gerbier, J. Morales, P. G. Picozza, W. Seidel, T. Sumner, M. Yamashita

\section{References}

[1] A. Cesarini et al. Astrop. Phys. 21, 267-285, 2004; [astro-ph/0305075] and references therein

[2] H. Mayer-Hasselwander et al., Astron. Astrophys. 335 (1998) 161

[3] P. Spillantini et al. At $24^{\text {th }}$ ICRC, OG 10.3.7 (1995); V. Bonvicini et al. NIM A461 (2001) 262

[4] W. Atwood et al. NIM A342 (1994) 302

[5] K. Tsuchiya et al. Astroph. J. 608 (2004) L115 and references therein

[6] F. A. Aharonian et al [Astro-ph/0408145] and references therein

[7] J. Cortina et al, Astroph. Space Sci 297 (2005) 245 
[8] K. Kosack et al. Astroph. J. 608 (2004) L97 and references therein

[9] SuperK, S. Desai et al. [Astro-ph/040425]

[10] [MACRO, M. Ambrosio et al. Phys. Rev. D 48 (1999) 08002; [astro-ph/0002492]

[11] BAKSAN, M. M. Boliev et al. 24 ${ }^{\text {th }}$ ICRC 1 (1995) 722

[12] AMANDA, J. Ahrens et al. Phys. Rev. D 66 (2002) 032006

[13] For a review see for example: N. Fornengo Nucl Phys. B (Proc. Suppl.) 110 (2002) 26; see also: Y. G. Kim et al. J. [hep-ph/0208069]

[14] J. Lindhard et al. Mat. Fys. Med. Dan. Vid. Sel. 33, n. 10 and n. 14 (1963)

[15] A. Drukier et al.; Phys. Rev. D33 (1986) 3495; K. Freese et al.; Phys. Rev. D37 (1988) 3388; M. Lewis and K. Freese; [astro-ph0307190]

[16] R. Bernabei et al. Riv. N.Cim. 26 n.1 (2003), IJMPD 13 (2004) 2127 and references therein

[17] LIBRA; R. Bernabei et al. [Astro-ph/0311046] and Nucl. Phys. B 138 (Proc. Suppl.) (2005) 48

[18] ANAIS; J. Morales et al.; Nucl. Phys. B (Proc. Suppl.) 118 (2003) 525

[19] CDMS04; D. S. Akerib et al. Phys. Rev. Let. 93 (2004) 211301

[20] CDMS 05; L. Baudis, these proceedings

[21] EDELWEISS; V. Sanglard [astro-ph/0503265]

[22] CRESST [astro-ph/0408006]

[23] C. Savage et al [astro-ph/0408346]

[24] A. Hitachi et al. Phys. Rev. B 27 (1983) 5279

[25] P. Benetti et al. Nucl. Instr. Meth.; A327 (1993) 203

[26] D. Cline et al. Astrop. Phys. 12 (2000), 377; see also: M. Yamashita et al. Astrop. Phys. 20 (2003) 79

[27] R. Bernabei et al. Phys. Lett. B436 (1998) 379; Phys. Lett. B465 (1999) 315

[28] ZEPLIN; G. J. Alner et al. Astrop. Phys. 23 (2005) 444

[29] XMASS; see e.g. S. Moriyama, $4^{\text {th }}$ Int. Workshop on Identif. of Dark Matter, Sept. 2002

[30] XENON; E. Aprile et al. [Astro-ph/0407575]; NSF proposal n. 0201740; LNGS LoI 33/03 (2003)

[31] R. Brunetti et al. astro-ph/0411491, 0405342; proposal: WARP.PV.INFN.IT/proposal.pdf; propaddendum.pdf 